Article

\title{
Preliminary Studies on an Innovative Bioactive Skin Soluble Beauty Mask Made by Combining Electrospinning and Dry Powder Impregnation
}

\author{
Jorge Teno ${ }^{1}\left(\mathbb{D}\right.$, María Pardo-Figuerez ${ }^{1,2, *\left(\mathbb{D}, \text { Nancy Hummel }^{3} \text {, Vincent Bonin }\right.}{ }^{3}$, \\ Alessandra Fusco ${ }^{4,5}$ (D) Claudio Ricci ${ }^{4}(\mathbb{D})$, Giovanna Donnarumma ${ }^{4,5}$, \\ Maria-Beatrice Coltelli ${ }^{4,6}\left(\mathbb{D}\right.$, Serena Danti ${ }^{6}(\mathbb{D})$ and Jose María Lagaron ${ }^{2, *(D)}$ \\ 1 R\&D Department, Bioinicia S.L., Calle Algepser 65 nave 3, Paterna, 46980 Valencia, Spain; \\ jteno@bioinicia.com \\ 2 Novel Materials and Nanotechnology Group, Institute of Agrochemistry and Food Technology (IATA), \\ Spanish Council for Scientific Research (CSIC), Calle Catedrático Agustín Escardino Benlloch 7, Paterna, \\ 46980 Valencia, Spain \\ 3 Fibroline SA, Swen Parc, Bât 4C, 1 rue des Vergers, 69760 Limonest, France; nhummel@fibroline.com (N.H.); \\ vbonin@fibroline.com (V.B.) \\ 4 Consorzio Interuniversitario Nazionale per la Scienza e Tecnologia dei Materiali (INSTM), \\ 50121 Florence, Italy; alessandra.fusco@unicampania.it (A.F.); ricciclaudio09@gmail.com (C.R.); \\ giovanna.donnarumma@unicampania.it (G.D.); maria.beatrice.coltelli@unipi.it (M.-B.C.) \\ 5 Department of Experimental Medicine, University of Campania “Luigi Vanvitelli", 80138 Naples, Italy \\ 6 Department of Civil and Industrial Engineering, University of Pisa, 56122 Pisa, Italy; serena.danti@unipi.it \\ * $\quad$ Correspondence: mpardo@iata.csic.es (M.P.-F.); lagaron@iata.csic.es (J.M.L.)
}

Received: 26 November 2020; Accepted: 9 December 2020; Published: 11 December 2020

check for updates

\begin{abstract}
The world of cosmetics is now aiming at biobased materials which are skin-compatible and can be used to generate more sustainable beauty masks with enhanced bioactivity. This work presents, in this line of interest, the combination of two innovative technologies, namely electrospinning and dry powder impregnation, to generate biobased skin soluble electrospun pullulan carriers dry impregnated with chitin nanofibrils-nanolignin-glycyrrethinic acid (CLA) complexes, as effective biobased and skin compatible beauty masks. The scalability of the pullulan electrospun carrier and bioactive complexes impregnation were optimized and the morphology evaluated. Subsequently, skin compatibility and mask effectiveness were investigated in vitro and in vivo. The results showed that cell viability was optimal for both impregnated and neat pullulan fibers. Additionally, the CLA impregnated pullulan fibers were able to upregulate the endogenous antimicrobial molecule HBD-2. Preliminary studies in vivo indicated that the beauty mask containing the CLA complexes significantly decreased area, length and depth of forehead and crow's feet wrinkles, and significantly increased moisturizing levels in the skin. The developed beauty mask was also seen to increase skin firmness, while it did not show skin irritation after the test. The work demonstrates that the combination of these two technologies may open new alternatives to more sustainable bioactive cosmetic products for the skin.
\end{abstract}

Keywords: pullulan; chitin nanofibril; glycyrrhetinic acid; nanolignin; antiaging

\section{Introduction}

The demand for effective skin care products has grown exponentially over the past few decades, mainly due to an overall increase in the age population along with a serious increase in air pollution [1]. The consumer has not only become more self-conscious of their own health, but also of their environment, 
since concerns about pollution have been made evident across the population with the world's current plastic waste crisis and the consequent circular economy strategies [2]. For this reason, it is not uncommon to find consumers choosing what they are buying by looking carefully at the ingredients of the products. This is particularly recurrent in the field of food, but also in cosmetics, where claims such as "bio", "green", or "natural" are popularly used $[3,4]$.

To meet consumer's demands, the cosmetic sector is searching alternatives to current materials by developing innovative formulations, in which effective skin products are based on natural and sustainable ingredients [5,6]. Generating functional biobased beauty masks has become one of the key aspects to investigate in the cosmetic industry, being the use of bio-based hydrophilic polymers to produce beauty masks a good alternative to conventional masks [1]. Among the many bio-based hydrophilic polymers, the use of polysaccharides, such as pullulan, may be a good option to generate efficient biobased beauty masks. Pullulan is a non-ionic water-soluble biopolymer produced using various substrates such as starch, distilled by-products or other residues $[7,8]$. The highly hydrophilic nature of this odorless material allows to instantaneously retain water when humidified and solubilizes very fast, thus appearing ideal to be used in contact with the skin. Consequently, the active ingredients are released immediately and waste generation is avoided [9]. Additionally, pullulan has been declared safe with a generally recognized as safe (GRAS) status by the Food and Drug Administration in the USA, as well as certified harmless for food usage by regulations in other countries so it can deem as acceptable for their applications in the cosmetic field $[7,10]$.

Electrospinning is an emerging technique which has been used in several fields such as pharma, biomedicine, food packaging, air filtration and cosmetics, with relatively high success $[9,11,12]$. When compared to conventional methods to generate non-woven structures (i.e., melt blown), electrospinning has various advantages such as its nano-scale fiber diameter, which enables a high-surface/volume ratio and the versatility of the technology, which enables the processing of a vast number of different materials [13]. The fibrous materials obtained from this technique appear as interconnected meshes of fibers with size approaching the nanoscale, which closely resembles the three-dimensional (3D) structure of the fibrous extracellular matrix (ECM). Such a structure can be very beneficial for skin care products, since a high skin contact and compatibility can be achieved due to the high surface area offered by the nanofibers and its 3D configuration [14]. In the cosmetic field, the use of electrospinning can be also used to overcome current issues related to the preservation of active ingredients by efficiently trapping them inside the fibers or within their mesh pores. Conventional beauty facial masks are made of non-woven structures that have previously been soaked with active ingredients. If preservatives are not used, these ingredients are not efficiently protected by a wet formulation approach. As such, sensitive ingredients like antioxidants can undergo degradation even before the mask is used, thus reducing their effectiveness. A dry state beauty mask would certainly increase the stability of the nutrients, minimizing their oxidation or degradation and avoiding the use of emulsifiers, preservatives, and other chemicals, further enhancing the sustainability of the mask $[7,14,15]$. However, certain active ingredients may poorly interact with the carrier mixture during electrospinning, hence negatively affecting the stability of the process, and ultimately leading to an inhomogeneous spatial distribution of the molecules in the tissue area. When this is the case, the availability of novel technologies able to impregnate porous materials with powders from the solid state, for example via high intensity alternating electric field, open the possibility of producing dry beauty masks based on nonwovens impregnated with bioactive fillers. Dry powder impregnation via electric field is a novel technology patented by Fibroline S.L. that allows the impregnation of powder form materials into porous structures (textiles, nonwovens, papers, foams, etc.) by applying an alternative electric field [16]. The specific concentration of an electrical field in different areas leads to a very homogenous distribution of the particles inside the substrate. In this case, compared to customarily used wet processes, dry impregnation technologies help to create new biofriendly products by avoiding the use of solvents, reducing pollution, and also reducing considerably energy consumption. 
Complexes of chitin nanofibrils-nanolignin-glycyrrethinic acid (CLA) have resulted promising for skin contact applications $[17,18]$. Chitin nanofibril $(\mathrm{CN})$ is a non-toxic crystalline polymer derived from crustacean waste which has shown excellent skin compatibility due to its high moisturizing effectiveness. Nanolignin (NL), a negatively charged by-product derived from forestry and agri-food waste, is reported to show beneficial properties to human health, for example, antioxidant and photoprotective activities due to its high phenolic content. The positively charged $\mathrm{CN}$ can interact with the negatively charged LN to form nanocomplexes which in turn are able to incorporate biomolecules in their structure, as it is the case of glycyrrhetinic acid (GA) [17,18]. This acid is extracted from the licorice plant and characterized by their antioxidative, anti-inflammatory and antimicrobial properties. The strong anti-inflammatory efficacy of this acid is particularly important, since inflammation processes are the start point of many skin issues, such as acne or atopic skin [9,19-21].

In this work, the dry impregnation technology has allowed to impregnate this type of complexes into electrospun non-woven pullulan fibers that perfectly embedded the microparticles without affecting the integrity of the electrospun structure. Therefore, this study reports the production and validation of a pullulan electrospun beauty mask impregnated with CLA micro complexes with the dry powder impregnation technology. The effectiveness of the beauty mask was demonstrated by in vitro and in vivo tests in healthy human volunteers. This study suggests that the combination of these two innovative technologies may open new alternatives to conventional beauty masks by generating effective cosmetic products which can in turn provide sustainable and biofriendly solutions.

\section{Materials and Methods}

\subsection{Materials}

The powder complex consisting of CN-NL-GA (also named as CLA) was supplied by Texol s.r.l. (Pescara, Italy). Pullulan (cosmetic grade) was supplied by Hayashibara Co., Ltd. (Okayama, Japan). Deionized water was employed for pullulan dissolution. Immortalized human keratinocytes, $\mathrm{HaCaT}$ cell line, were obtained from ATCC-LGC Standards (Milan, Italy). Human mesenchymal stromal cells (HMSCs) were supplied from Merck Millipore S.A.S. (Burlington, MA, USA). $\mathrm{MgCl}_{2}$, Dulbecco's Modified Essential Medium (D-MEM), L-glutamine, penicillin, streptomycin, and fetal calf serum were purchased from Invitrogen (Carlsbad, CA, USA). AlamarBlue and Calcein AM were bought from ThermoFisher Scientific (Waltham, MA, USA). LC Fast Start DNA Master SYBR Green kit was obtained from Roche Applied Science (Euroclone S.p.A., Pero, Italy). Dulbecco's phosphate-buffered saline (DPBS) was provided by Sigma-Aldrich (Milan, Italy). StemMACS ${ }^{\mathrm{TM}}$ Adipodiff medium was obtained from Miltenyi Biotech s.r.l. (Bologna, Italy). Sodium Lauryl Sulphate (SLS) was supplied by Sigma Aldrich (Madrid, Spain).

\subsection{Solution Preparation and Characterization}

Pullulan solution was prepared in an aqueous solution with a $21 \mathrm{wt} . \%$ pullulan, heated at $37^{\circ} \mathrm{C}$ and stirred at $450 \mathrm{rpm}$ overnight. The physicochemical properties of the solution were analyzed in terms of viscosity, conductimetry and surface tension.

The apparent viscosity ( $\eta$ a) was determined using a rotational viscosity meter Visco BasicPlus L from Fungilab S.A. at $50 \mathrm{rpm}$ with a L3 spindle (San Feliu de Llobregat, Spain). The surface tension was measured using a DynoTester tensiometer from Krüss GmbH (Hamburg, Germany). The conductivity was evaluated using a conductivity meter Seven2Go ${ }^{\mathrm{TM}}$ from Metler Toledo 742-ISM (Schwerzenbach, Switzerland). Measurements were carried out at room temperature.

\subsection{Electrospinning Pullulan Fiber Meshes}

The pullulan fiber meshes were prepared using a Fluidnatek ${ }^{\mathrm{TM}}$ LE-500 production equipment with an industrial $50 \mathrm{~cm}$ wide roll-to-roll system from Bioinicia S.L. (Valencia, Spain), shown in Figure 1a. The solutions were pumped through a linear multi needle injector at a feed rate of $0.77 \mathrm{~mL} / \mathrm{h}$ 
per needle, applying a voltage of $+40 \mathrm{kV}$ on the emitter and a voltage of $-30 \mathrm{kV}$ on the collector. The fibers were collected at a distance of $32.5 \mathrm{~cm}$ over black polyethylene (PE) substrate at a speed rate of $10 \mathrm{~mm} / \mathrm{s}$, under controlled environmental conditions of $20{ }^{\circ} \mathrm{C}$ and $30 \%$ relative humidity (RH). The deposition time was optimized and adjusted accordingly to obtain samples with a surface density of approximately $20 \mathrm{~g} / \mathrm{m}^{2}$ (Figure $1 \mathrm{~b}$ ).

a)

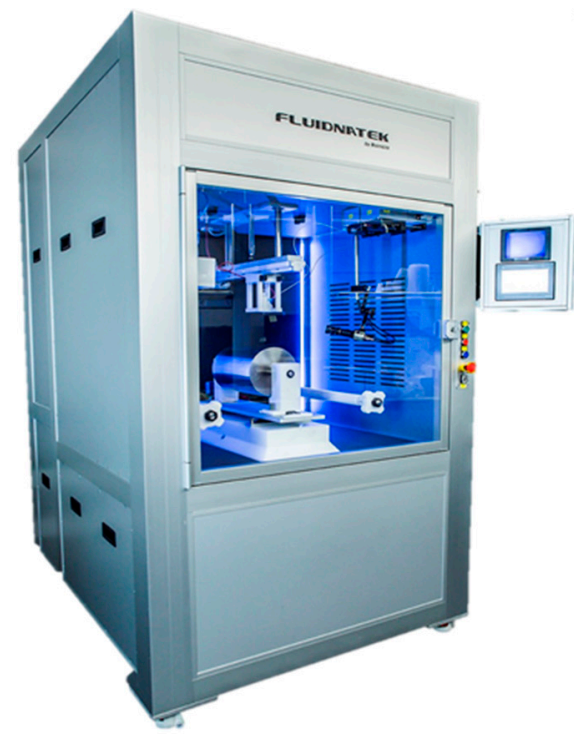

b)

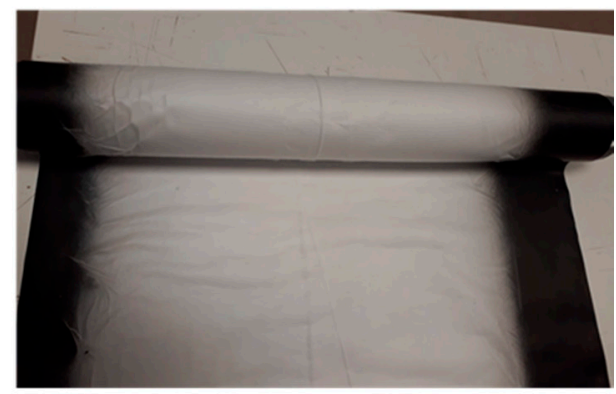

Figure 1. (a) Electrospinning equipment Fluidnatek ${ }^{\mathrm{TM}}$ LE-500; (b) Electrospun pullulan mesh rolled sample.

\subsection{Impregnation of Electrospun Mesh}

Electrospun pullulan meshes were impregnated with CLA using the dry powder impregnation technology (Fibroline S.A., Lyon, France) as illustrated in Figure 2. Briefly, the samples were inserted in the impregnation chamber. Then, the engraved sleeve containing the powder was applied onto the pullulan mesh and the alternating electric field allowed the impregnation of the powder across the porosities of the non-woven pullulan material. The voltage applied to transfer the particles from the sleeve to the pullulan substrate was $40 \mathrm{kV}$ and the rolls of material contained $3.5 \mathrm{wt} \%$ of CLA complexes. The process of impregnating the pullulan carrier sample has been illustrated throughout Figure 2a-c.

a)

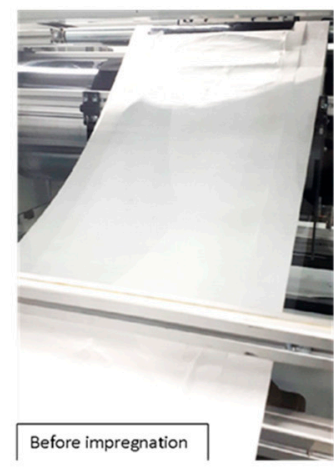

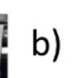
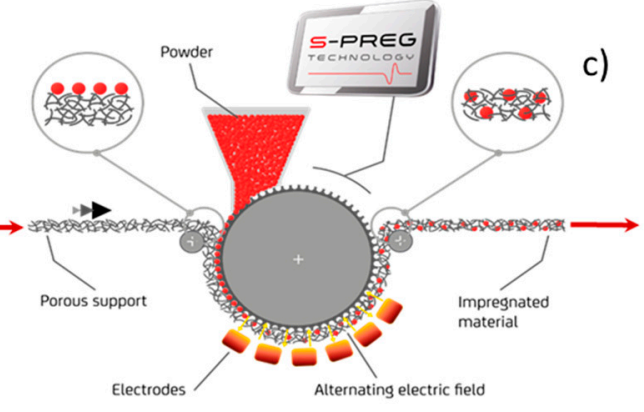

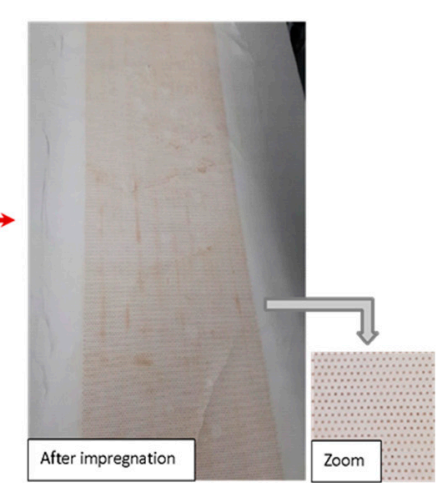

Figure 2. (a) Pullulan electrospun mesh being inserted into the pilot equipment for dry impregnation. (b) Sketch of the dry impregnation technology (S-Preg). (c) Pullulan electrospun mesh impregnated with CLA complexes. 


\subsection{Beauty Mask Production and Characterization}

The fiber morphology was analyzed by Field Emission Scanning Electron Microscopy (FE-SEM) using a Hitachi S-4800 (Hitachi High Technologies Corp., Tokyo, Japan), with an electron beam acceleration of $10 \mathrm{kV}$. The samples for SEM analysis were previously sputtered with a gold-palladium mixture for $2 \mathrm{~min}$. Fiber diameter was determined using ImageJ Software (version 1.52, National Institutes of Health, Bethesda, MD, USA). Average fiber diameter was assessed on at least 100 fibers randomly selected from the software.

The particle size distribution of the CLA complexes was measured with a Mastersizer 2000, (Malvern Instruments, Ltd., Worcestershire, UK) and the impregnated samples were observed via FE-SEM (FEI FEG-Quanta 450 instrument Field Electron and Ion Company, Hillsboro, OR, USA).

Chemical composition of the beauty masks was evaluated by Fourier transform infrared spectroscopy (FT-IR) using a Nicolet T380 instrument (ThermoFisher Scientific, Waltham, MA, USA) equipped with a Smart ITX Attenuated Total Refraction (ATR) accessory with diamond plate (ThermoFisher Scientific, Waltham, MA, USA).

Beauty mask samples prototypes used for in vivo studies were cut employing a Laser pro Spirit LS laser cut equipment. The size of the mask was approximately $22 \mathrm{~cm}$ length $\times 19 \mathrm{~cm}$ width with weighs of approximately $0.5-0.6 \mathrm{~g}$.

\subsection{In Vitro Skin Compatibility}

Skin compatibility of the beauty masks was investigated in vitro using human dermal keratinocytes (HaCaT cells) to represent epidermal and human mesenchymal stromal cells (hMSCs) to represent connective (i.e., dermal/hypodermal) layers. Each material sample was sterilized overnight via UV irradiation. HaCaT cells were cultured in $75 \mathrm{~cm}^{2}$ tissue culture flasks using D-MEM supplemented with $1 \%$ penicillin-streptomycin, $1 \%$ L-glutamine and $10 \%$ fetal calf serum in a humidified incubator set at $37{ }^{\circ} \mathrm{C}$ in $95 \%$ air and $5 \% \mathrm{CO}_{2}$. When $80 \%$ confluence was reached, the cells were replated in 12 -well plates at a seeding density of $10^{5}$ cells/well. The materials $\left(1 \mathrm{~cm}^{2} \mathrm{each}\right)$ were inserted in the wells containing HaCaT cells for $6 \mathrm{~h}$ and $24 \mathrm{~h}$.

The immunomodulatory properties of pullulan and pullulan/CLA fiber were assayed via real time reverse transcriptase polymer chain reaction (qRT-PCR). At the timepoints, the mRNA was extracted from the cells and the levels of expression of a panel of cytokines involved in inflammatory response was evaluated. Specifically, the pro-inflammatory interleukins (ILs) IL-1 $\alpha$, IL-1 $\beta$, IL-6, IL-8, and tumor necrosis factor alpha (TNF- $\alpha$ ), as well as the anti-inflammatory cytokine transforming growth factor beta (TGF- $\beta$ ) and the antimicrobial peptide human beta defensin 2 (HBD-2) were evaluated. Briefly, the total RNA was isolated with TRizol, and $1 \mu \mathrm{m}$ of RNA was reverse-transcribed into complementary DNA (cDNA) using random hexamer primers at $42{ }^{\circ} \mathrm{C}$ for $45 \mathrm{~min}$, according to the manufacturer's instructions. PCR was carried out with the LC Fast Start DNA Master SYBR Green kit using $2 \mu \mathrm{L}$ of cDNA, corresponding to $10 \mathrm{ng}$ of total RNA in a $20 \mu \mathrm{L}$ final volume, $3 \mathrm{mM} \mathrm{MgCl}$, and $0.5 \mu \mathrm{M}$ sense and antisense primers (Table 1). The results were normalized by the expression of the same cytokine in untreated cells, as a control. 
Table 1. qRT-PCR parameters utilized to analyze the immunomodulatory properties of the material. The table indicates the gene analyzed, the primer sequences, operational conditions, and product size.

\begin{tabular}{|c|c|c|c|}
\hline Gene & Primers Sequence & Conditions & Product Size (bp) \\
\hline \multirow{2}{*}{ IL-1 $\alpha$} & 5'-CATGTCAAATTTCACTGCTTCATCC-3' & \multirow{2}{*}{$\begin{array}{l}5 \mathrm{~s} \text { at } 95^{\circ} \mathrm{C}, 8 \mathrm{~s} \text { at } 55^{\circ} \mathrm{C}, \\
17 \mathrm{~s} \text { at } 72^{\circ} \mathrm{C} \text { for } 45 \text { cycles }\end{array}$} & \multirow{2}{*}{421} \\
\hline & 5'-GTCTCTGAATCAGAAATCCTTCTATC-3' & & \\
\hline \multirow{2}{*}{ IL-1 $\beta$} & 5'-GCATCCAGCTACGAATCTCC-3' & \multirow{2}{*}{$\begin{array}{l}5 \mathrm{~s} \text { at } 95^{\circ} \mathrm{C}, 14 \mathrm{~s} \text { at } 58^{\circ} \mathrm{C}, \\
28 \mathrm{~s} \text { at } 72^{\circ} \mathrm{C} \text { for } 40 \text { cycles }\end{array}$} & \multirow{2}{*}{708} \\
\hline & $5^{\prime}$-CCACATTCAGCACAGGACTC-3' & & \\
\hline \multirow{2}{*}{ IL-6 } & 5'-ATGAACTCCTTCTCCACAAGCGC-3' & \multirow{2}{*}{$\begin{array}{l}5 \text { s at } 95^{\circ} \mathrm{C}, 13 \text { s at } 56{ }^{\circ} \mathrm{C}, \\
25 \text { at } 72{ }^{\circ} \mathrm{C} \text { for } 40 \text { cycles }\end{array}$} & \multirow{2}{*}{628} \\
\hline & 5'-GAAGAGCCCTCAGGCTGGACTG-3' & & \\
\hline \multirow{2}{*}{ IL-8 } & 5'-ATGACTTCCAAGCTGGCCGTG-3' & \multirow{2}{*}{$\begin{array}{l}5 \mathrm{~s} \text { at } 94^{\circ} \mathrm{C}, 6 \mathrm{~s} \text { at } 55^{\circ} \mathrm{C}, \\
12 \mathrm{~s} \text { at } 72{ }^{\circ} \mathrm{C} \text { for } 40 \text { cycles }\end{array}$} & \multirow{2}{*}{297} \\
\hline & 5'-TGAATTCTCAGCCCTCTTCAAAAACTTCTC-3' $^{\prime}$ & & \\
\hline \multirow{2}{*}{ TNF- $\alpha$} & 5'-CAGAGGGAAGAGTTCCCCAG-3' & \multirow{2}{*}{$\begin{array}{l}5 \text { s at } 95^{\circ} \mathrm{C}, 6 \text { s at } 57^{\circ} \mathrm{C} \text {, } \\
13 \text { s at } 72^{\circ} \mathrm{C} \text { for } 40 \text { cycles }\end{array}$} & \multirow{2}{*}{324} \\
\hline & 5'-CCTTGGTCTGGTAGGAGACG-3' & & \\
\hline \multirow{2}{*}{ TGF- $\beta$} & 5'-CCGACTACTACGCCAAGGAGGTCAC-3' & \multirow{2}{*}{$\begin{array}{l}5 \mathrm{~s} \text { at } 94^{\circ} \mathrm{C}, 9 \mathrm{~s} \text { at } 60^{\circ} \mathrm{C}, \\
18 \mathrm{~s} \text { at } 72^{\circ} \mathrm{C} \text { for } 40 \text { cycles }\end{array}$} & \multirow{2}{*}{439} \\
\hline & 5'-AGGCCGGTTCATGCCATGAATGGTG-3' & & \\
\hline HBD-2 & 5'-GGATCCATGGGTATAGGCGATCCTGTTA-3' & $\begin{array}{l}5 \mathrm{~s} \text { at } 94^{\circ} \mathrm{C}, 6 \mathrm{~s} \text { at } 63^{\circ} \mathrm{C}, \\
10 \mathrm{~s} \text { at } 72^{\circ} \mathrm{C} \text { for } 50 \text { cycles }\end{array}$ & 198 \\
\hline
\end{tabular}

Non-differentiated hMSCs were cultured in growth medium in presence of beauty mask samples, which solubilized inside for 3 days. Adipogenic differentiation of hMSCs was tested in vitro using pullulan and pullulan/CLA pre-treated cell samples, which were cultured in Adipodiff medium for 5 days. At the endpoint, the adipogenic pre-differentiated hMSCs were incubated with Calcein $\mathrm{AM} / \mathrm{DPBS}$ solution, as from the manufacturer's instructions, for $20 \mathrm{~min}$ at $37^{\circ} \mathrm{C}$ in the dark, and observed under a FITC filter equipped inverted microscope (Nikon Eclipse CI; Nikon Instruments, Amsterdam, The Netherlands) with a digital camera.

At the abovementioned timepoints, the AlamarBlue test was performed on $\mathrm{HaCaT}$ cells, undifferentiated hMSCs and adipogenic pre-differentiated hMSCs. The test was pursued following the manufacturer's protocol after $4 \mathrm{~h}$ incubation with the dye solution. AlamarBlue incorporates a REDOX indicator that changes color according to cell metabolic activity. The supernatants were read with a spectrophotometer (Victor3; PerkinElmer, Waltham, MA, USA) in a 96 well plate, using a double wavelength reading at $570 \mathrm{~nm}$ and $600 \mathrm{~nm}$. Finally, the reduced percentage of the dye (\%ABred) was calculated by correlating the absorbance values and the molar extinction coefficients of the dye at the selected wavelengths, as from the equation provided by the manufacturer. The equation applied is shown below, in which: $\lambda=$ absorbance, $\mathrm{s}=$ sample, and $\mathrm{c}=$ control:

$$
\% \mathrm{AB}_{\text {red }}=100 \cdot \frac{\left(117,216 \cdot \lambda_{\mathrm{s}(570 \mathrm{~nm})}-80,586 \cdot \lambda_{\mathrm{s}(600 \mathrm{~nm})}\right)}{\left(155,677 \cdot \lambda_{\mathrm{c}(600 \mathrm{~nm})}-14,652 \cdot \lambda_{\mathrm{c}(570 \mathrm{~nm})}\right)}
$$

\subsection{In Vivo Skin Compatibility}

\subsubsection{Inclusion and Exclusion Criteria}

The study was carried out according to the Declaration of Helsinki as revised successively in Seoul (Ethical Principles for Medical Research Involving Human Subjects) [22], after the approval of the local ethics Committee (Comité de ética de la investigación con medicamentos, La Fe, Spain; approval number FPNT-CEIB-04(B)). A total of 30 healthy female volunteers were recruited according to the following general inclusion and exclusion criteria described in Table 2. 
Table 2. Inclusion and exclusion criteria for the human panel study on beauty masks.

\begin{tabular}{|c|c|}
\hline General Inclusion Criteria & General Exclusion Criteria \\
\hline $\begin{array}{l}\text { Presence of wrinkles in forehead and crow's } \\
\text { feet areas. }\end{array}$ & $\begin{array}{l}\text { Allergy or reactivity to some of the components of the } \\
\text { product, or a product with similar category than tested one. }\end{array}$ \\
\hline $\begin{array}{l}\text { Relative frequency in the use of skin care } \\
\text { cosmetic products }\end{array}$ & Surgery at the facial area. \\
\hline $\begin{array}{l}\text { Age: } 30-65 \text { years. } \\
\text { Gender: Female. }\end{array}$ & $\begin{array}{l}\text { Relevant cutaneous marks in the experimental areas, which } \\
\text { could interfere with the measurements (scars, sunburns, etc.). }\end{array}$ \\
\hline Skin phototype (Fitzpatrick): II, III and IV. & In-use relevant pharmacological or hormonal treatment. \\
\hline Skin type: Dry, combination and oily. & Presence of skin diseases or melanomas. \\
\hline $\begin{array}{l}\text { Last participation in clinical study, at least one } \\
\text { month before the start of this experiment. }\end{array}$ & \multirow{2}{*}{$\begin{array}{l}\text { Forecast of change of routine or relevant way of life, } \\
\text { during the period of study. }\end{array}$} \\
\hline $\begin{array}{l}\text { Understanding and signature of } \\
\text { Informed Consent. }\end{array}$ & \\
\hline
\end{tabular}

\subsubsection{In Vivo Effectiveness Assessment}

In order to board the in vivo assessment, the beauty mask (PBSK-1; referred as the electrospun pullulan carrier mask impregnated with CLA complexes) was compared with placebo mask (PBSK-2; referred as the electrospun pullulan carrier mask without active complexes impregnation). Two groups of 15 volunteers were recruited and underwent topical treatment during 4 weeks with mask PBSK-1 or mask PBSK-2. The masks were applied in the wet facial area for 10-15 min of application, once per week.

During the time course of the experiment, this is before (on day $D_{0}$ ), after 15 days $\left(D_{15}\right)$ and 30 days of treatment $\left(\mathrm{D}_{30}\right)$, images were taken with a Bio3D Structured-light Scanner (Bionos Biotech, Valencia, Spain) from the areas of the forehead (glabellar lines) and crow's feet (periocular wrinkles). Images were processed through specific software by generating 3D reconstructions from 2D images of the volunteers. Examples of this methodology is shown in Figure 3. From the 3D reconstruction, a region of interest is analyzed. In this case, the total area, length and depth of forehead wrinkles and crow's feet were then assessed. As well as this, skin firmness, moisturizing and TEWL levels were assessed through Cutometer ${ }^{\circledR}$ MPA 580, Corneometer ${ }^{\circledR}$ CM 825 and Tewameter ${ }^{\circledR}$ TM 300, respectively (Bionos Biotech). Anti-irritating activity (i.e., erythema levels) was also quantified after 5 days of treatment (daily) on forearms, using Mexameter ${ }^{\circledR}$, after inducing a mild irritation with SLS water solution at $3 \%(w / v)$. The irritated area was measured $1 \mathrm{~h}$ after removal of SLS patch (at time $\mathrm{T}_{1}$ ) and was then topically treated with mask PBSK-1 or mask PBSK-2 (twice per day, right or left arm), and measured every $24 \mathrm{~h}$ (at times $\mathrm{T}_{2}, \mathrm{~T}_{3}$, and $\mathrm{T}_{4}$ ). The mean values obtained by analyzing the 15 volunteers were normalized to their initial basal values (at time $\mathrm{T}_{0}$ ).

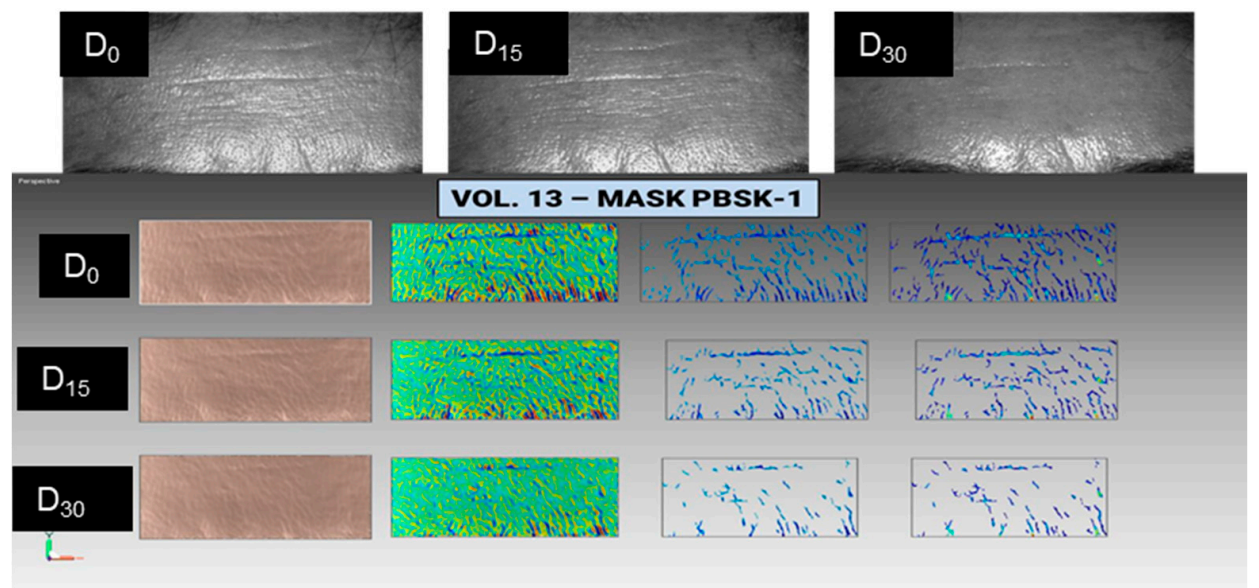

Figure 3. Example of wrinkle study carried out using the image analysis methodology reported above. 


\subsection{Statistical Analysis}

For in vitro studies, statistical analyses were carried out by SPSS software (SPSS v.16.0; IBM Corp., Chicago, IL, USA). All data were analyzed using a one-way analysis of variance (ANOVA).

For the in vivo studies, up to five repeated measurements of each parameters were taken at each timepoints $\left(D_{0}, D_{15}, D_{30}\right)$. Each technical value at each of the timepoints was normalized to the mean value of the repeated measurements at day 0 , being each volunteer its own control. Data were statistically analyzed applying a paired student T-test.

For both studies, probability $(p)$ values $<0.05$ were considered as statistically significant differences.

\section{Results}

\subsection{Beauty Mask Preparation and Characterization}

Based on previous results from the literature [23,24], an aqueous solution of $21 \mathrm{wt}$ \% of pullulan was optimized for upscaling purposes and the physicochemical properties of the solution were measured (see Table 3). The solution presented a high surface tension and slightly high conductivity, most likely due to the presence of water. On the other hand, a high viscosity was obtained due to a large polymer concentration necessary to generate the pullulan fibers.

Table 3. Physicochemical properties of the solution.

\begin{tabular}{cc}
\hline Property & Measurement \\
\hline Conductivity & $41.34 \mu \mathrm{S} / \mathrm{m}$ \\
Surface tension & $81.9 \mathrm{mN} / \mathrm{m}$ \\
Viscosity & $3249.3 \mathrm{cP}$ \\
\hline
\end{tabular}

Water-based solutions are commonly difficult to electrospin and consequently tedious to upscale [23]. In this case, the manufacture of pullulan electrospun carrier was successfully stable with a multi-needle system and was performed under a tight control of temperature and relative humidity. Figure $4 \mathrm{a}$ shows a representative SEM image of the pullulan electrospun mesh, showing a smooth and homogeneous fiber morphology with an average fiber diameter of $1.13 \pm 0.29 \mu \mathrm{m}$. The fiber distribution of the pullulan mesh has been presented in Figure $4 \mathrm{~b}$.

a)

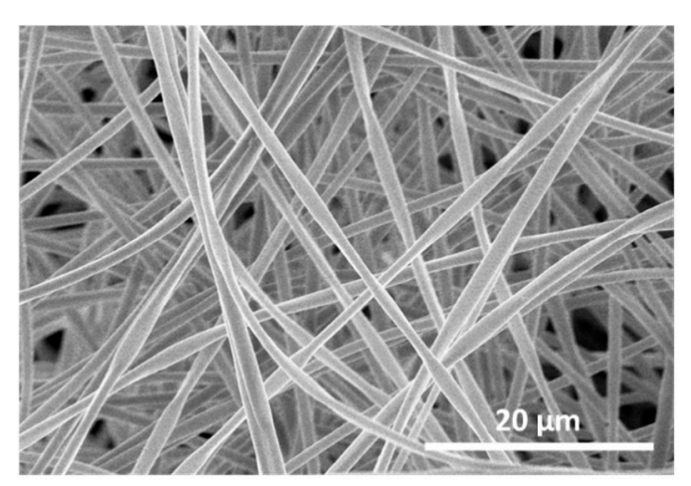

b)

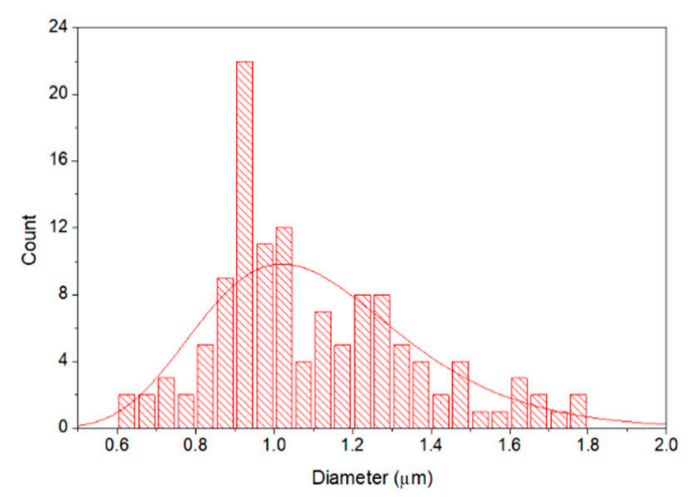

Figure 4. (a) Representative scanning electron microscope (SEM) images of the pullulan electrospun carrier (2200×); (b) Electrospun pullulan fiber size distribution. Scale bar is 20 microns.

Previous to CLA impregnation onto the pullulan meshes, the size of the particles was measured. As observed in Figure 5, the resultant particle size of the complexes was below $10 \mu \mathrm{m}(\mathrm{d} 90=3 \mu \mathrm{m})$, which was optimal to obtain an efficient powder impregnation. 


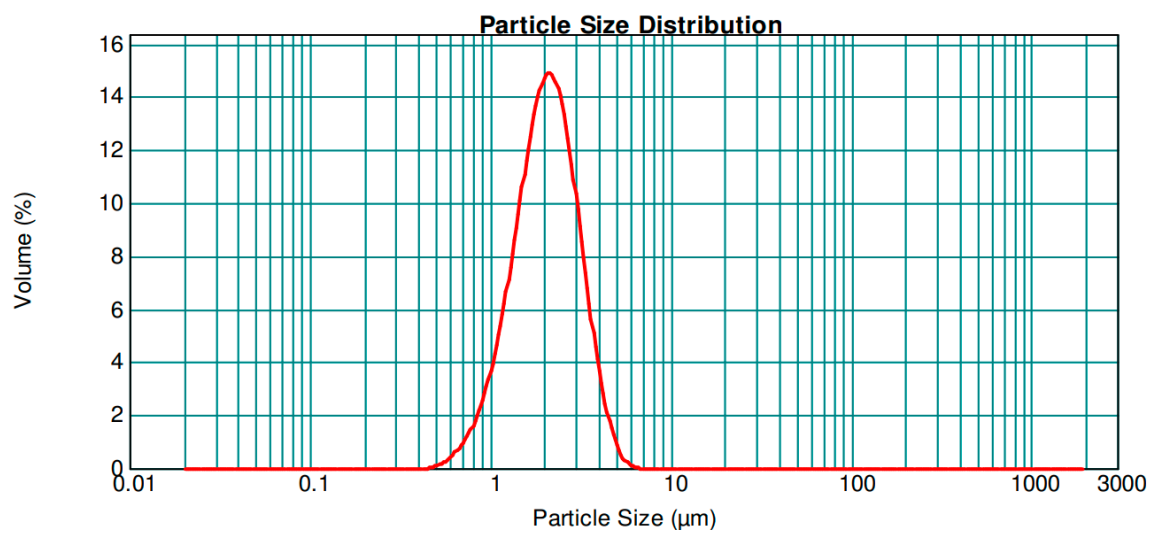

Figure 5. Particle size distribution of the CLA complexes. [CLA stands for CN-NL-GA].

A quantity of $0.7 \mathrm{~g} / \mathrm{m}^{2}$ of CLA was then deposited into the pullulan electrospun carrier. The upscaling of the impregnation onto the pullulan fibers showed good stability of the CLA spreading and dosing in each and across different batches. The clean white electrospun mat (Figure 2a) acquired an "earthy-like" color after impregnation (Figure 2c). At the microscopic level, the resultant impregnated material presented a homogeneous distribution of the complexes within the pullulan fibers, as observed in Figure 6.

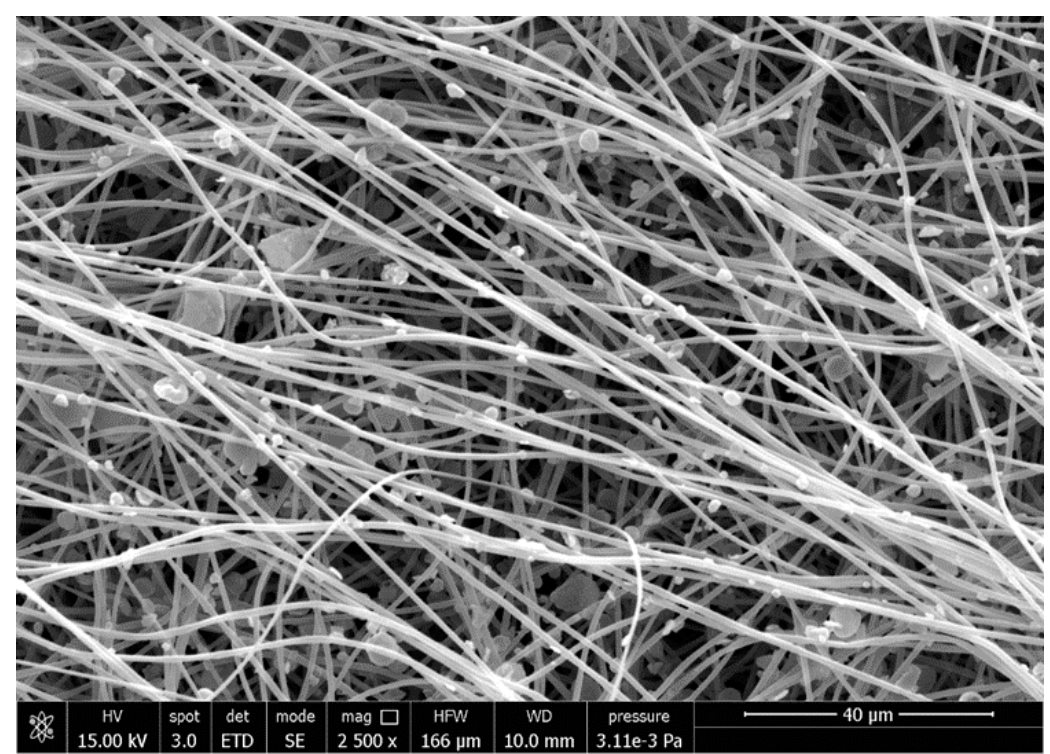

Figure 6. Representative SEM micrograph (2500×) of electrospun pullulan mesh impregnated with CLA, visible as microparticles attached to the fibers. [CLA stands for CN-NL-GA]. Scale bar is 40 microns.

The pullulan/CLA samples were characterized by ATR-FTIR spectroscopy, allowing the spectra of the sample surface to be analyzed. The obtained spectrum (Figure 7a) shows the main bands at $1006 \mathrm{~cm}^{-1}, 1077 \mathrm{~cm}^{-1}$ and $1147 \mathrm{~cm}^{-1}$. These bands are attributable at $\mathrm{C}-\mathrm{O}$ and $\mathrm{C}-\mathrm{C}$ bonds and deformational vibrations of the C-C-H, C-O-H and H-C-O bonds in the pullulan spectrum, as reported by several authors [25-27]. In fact, the spectrum of the pure pullulan powder, reported on Figure 7c, resulted very similar to the one of the pullulan/CLA, reported in Figure 7a. However, additional bands at $1620 \mathrm{~cm}^{-1}, 1561 \mathrm{~cm}^{-1}, 1342 \mathrm{~cm}^{-1}$ and $840 \mathrm{~cm}^{-1}$ were clearly noticed. These bands can be attributed to the CLA complexes, which are reported in Figure $7 \mathrm{~b}$. It is possible to observe the presence of the active complex because its content on the mask surface is high, thanks to the exceptionally low density of the electrospun nanostructured tissue. 


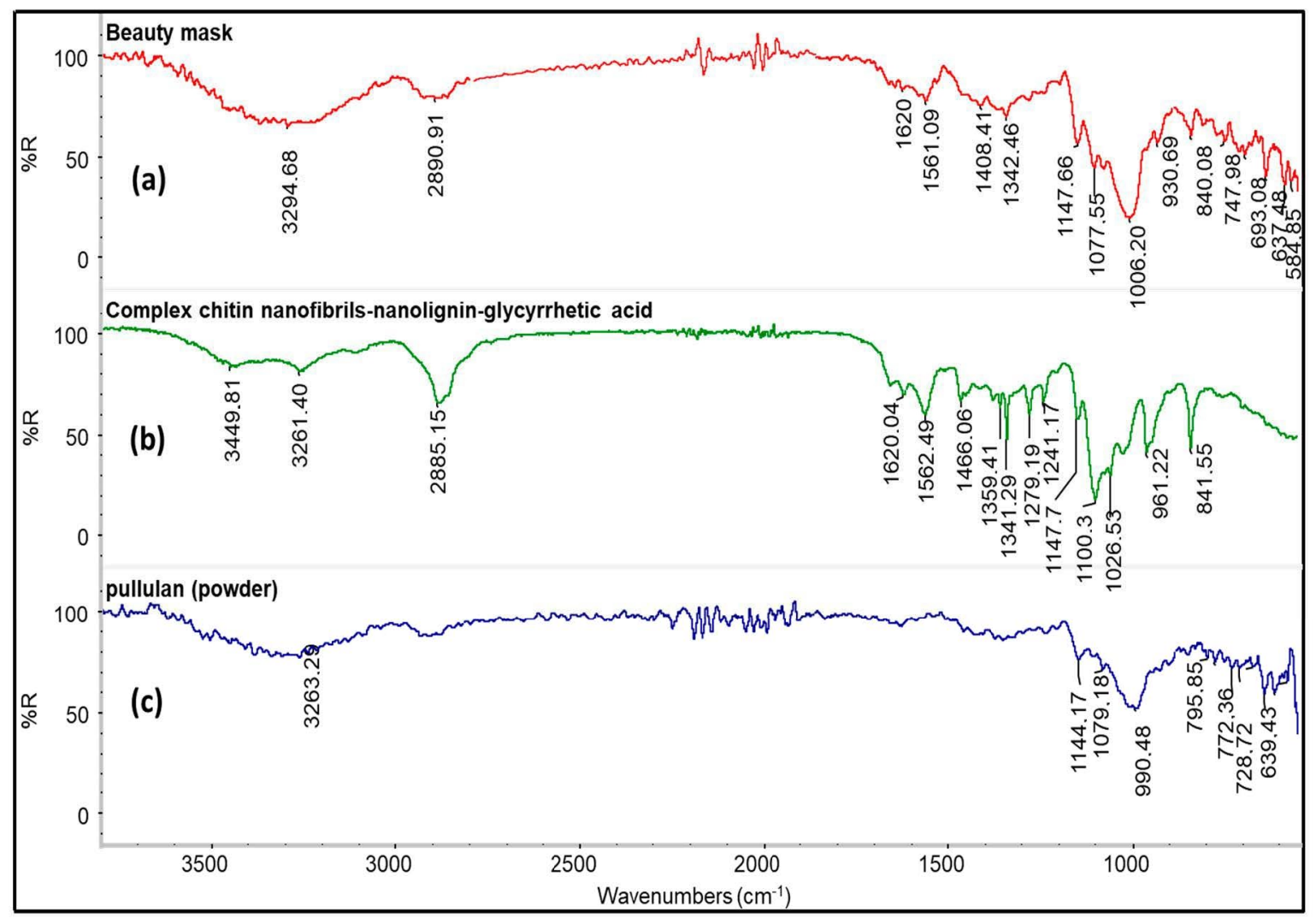

Figure 7. ATR-FTIR spectra of (a) pullulan/CLA (i.e., beauty mask); (b) CLA complex; (c) pure pullulan powder. [CLA stands for CN-NL-GA].

\subsection{In Vitro Compatibility Study}

The results of AlamarBlue reduction $\left(\mathrm{AB}_{\text {red }} \%\right)$, which identifies cell metabolic activity for the different cell types and timepoints are reported in Figure 8. The outcomes showed that cell viability, intended as metabolically active cells, was good and similar in CLA-impregnated pullulan and pure pullulan meshes. In fact, no statistically significant difference was observed between the samples belonging to the same groups. A remarkably favorable effect was observed with human dermal keratinocytes $\mathrm{HaCaT}$ cells. It has to be mentioned that different cell types may have different metabolic activity, especially primary cells like hMSCs. In addition, considering undifferentiated hMSCs, $\mathrm{AB}_{\mathrm{red}} \%$ increased over time, which indicates a good response of the cells to the dissolved biomaterials.

By exposing pullulan and pullulan/CLA fiber meshes to HaCaT cells, the expression of different pro- and anti-inflammatory cytokines, as well as the antimicrobial peptide HBD-2, were investigated at $6 \mathrm{~h}$ and $24 \mathrm{~h}$ using qRT-PCR. The results are reported in Figure 9.

Besides IL-6 and IL-8, pullulan fiber meshes are able to decrease some important pro-inflammatory cytokines, such as IL-1 (both $\alpha$ and $\beta$ isoforms), and most of all, they can upregulate the expression of the anti-inflammatory cytokine TGF- $\beta$. Therefore, they are endowed with mild anti-inflammatory properties. After CLA impregnation, the behavior changed to mild pro-inflammatory. Most importantly, only pullulan/CLA was able to strongly upregulate the endogenous antimicrobial molecule HBD-2.

After pullulan and pullulan/CLA administration in culture, hMSCs were differentiated into adipoblasts to represent the hypodermal layer of the skin. The hMSCs successfully differentiated by showing a roundish morphology with fatty vacuoles in the cytoplasm (arrows in Figure 10a). Moreover, the adipo-differentiated hMSCs were viable, as confirmed by Calcein AM fluorescent staining on live cells (Figure 10b,c). 


\section{Cell metabolic activity}

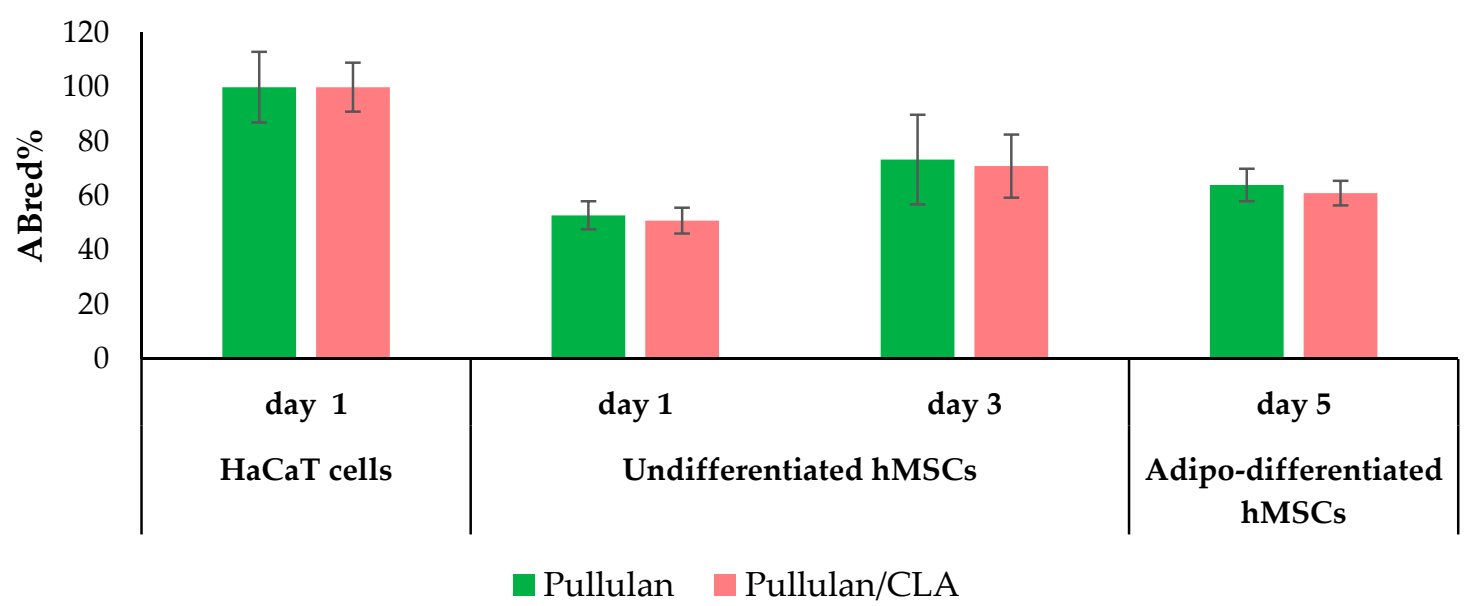

Figure 8. Bar graph showing ABred\%, which indicates metabolic activity of cells after being added during the culture with a piece of soluble beauty mask, either plain or CLA-impregnated pullulan fiber meshes. No statistically significant differences were observed between pullulan and pullulan/CLA in the same cell/timepoint groups ( $p=$ n.s.) [CLA stands for CN-NL-GA].
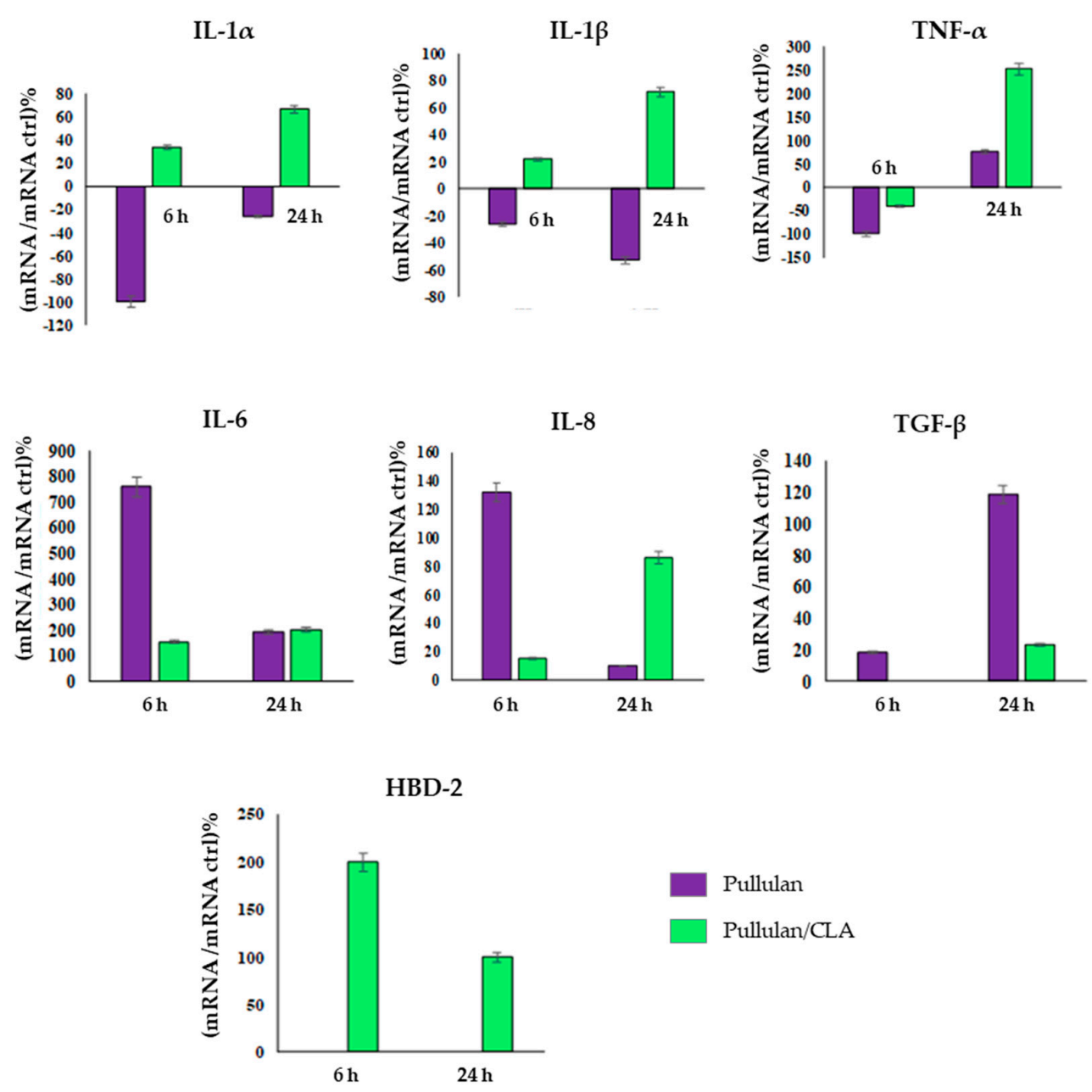

Figure 9. Relative gene expression of HBD-2 by HaCaT cells in contact with pullulan and pullulan/CLA (beauty mask) for $6 \mathrm{~h}$ and $24 \mathrm{~h}$. Data are reported as mean $\pm \mathrm{SD}$ and are expressed as percentage of increment relative to untreated $\mathrm{HaCaT}$ cells (as controls; ctrl). [CLA stands for CN-NL-GA]. 
(a)

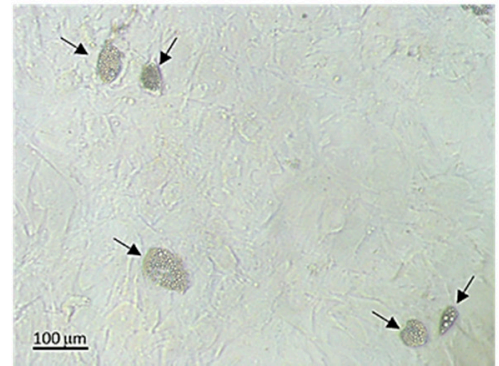

(b)

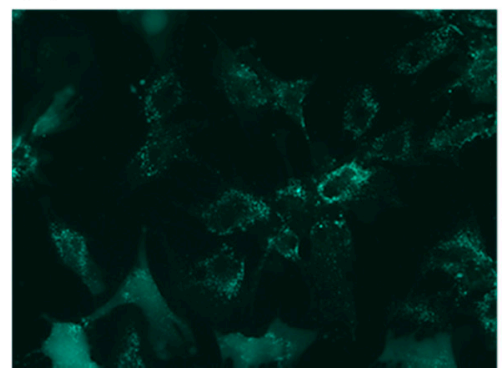

(c)

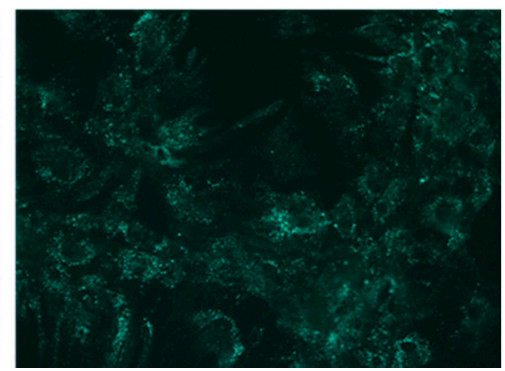

Figure 10. Adipo-differentiated hMSCs: (a) as observed under light microscopy: fat vesicles are pointed by arrows; (b,c) Live cell fluorescent staining (Calcein AM) of adipo-differentiated hMSCs placed together with (b) pullulan and (c) pullulan/CLA beauty mask samples for 5 days. Original magnification 100×. [CLA stands for CN-NL-GA].

\subsection{In Vivo Compatibility Study}

Preliminary to in vivo efficiency studies, an irritation test on human volunteers was carried out in order to dismiss any irritation issue. Figure 11 shows the plotted results showing that after effectively inducing a mild skin irritation on forearms, no significant differences in erythema levels were yielded upon application of mask PBSK-1 or mask PBSK-2, at none of the tested timepoints, thus confirming the suitability of the product for skin applications.

\section{Erythem a}

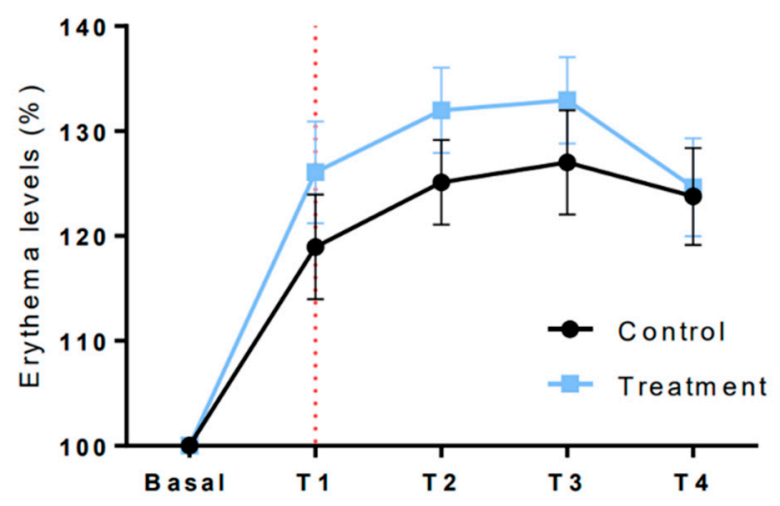

Figure 11. Functional analysis of the anti-irritant effects of mask PBSK-1 and mask PBSK-2 over time. The mean and the standard error of the median are plotted as percentage. A dashed red line is set at $\mathrm{T}_{1}$ and is indicative of the beginning of each treatment. For statistical significance, a paired t-test was applied to the normalized values of each time point, comparing the treated vs. untreated areas.

The in vivo results also confirmed the activity shown by the in vitro studies. Antiaging properties were assessed by measuring the total area, depth and length in every winkle at day 0, 15 and 30 . When the wrinkles in the forehead were assessed, results showed that treatment with mask PBSK-1 during 2 weeks (i.e., 2 applications) significantly decreased area and length of forehead wrinkles by $14.5 \% \pm 5.8 \%$ and $15.8 \pm 16.8 \%$, respectively. Differently, the treatment with mask PBSK-2 (placebo) did not show any statistically significant effect, comparing each volunteer with its own control at day 0 (Figure 12a,b). In the same way, the treatment with mask PBSK-1 during four weeks (i.e., four applications) significantly decreased the area, length, and depth of forehead wrinkles by $20.9 \% \pm 7.4 \%, 21.3 \% \pm 7.0 \%$, and $6.4 \% \pm 1.4 \%$, respectively. Also, in this case, the treatment with mask PBSK-2 did not show any statistically significant effect. Overall, comparison results between PBSK-1 and PBSK-2 indicated that the treatment with PBSK-1 significantly decreased area, length and depth of forehead wrinkles after 4 weeks of treatment (Figure 12a-c). 

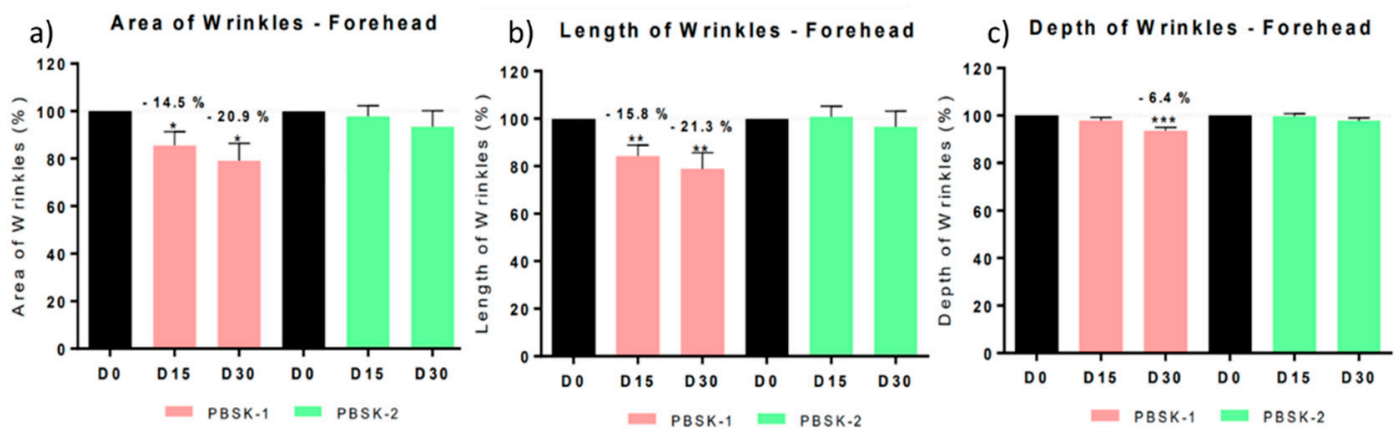

Figure 12. Graphical representation of the total area (a), length (b) and depth (c) of forehead wrinkles before $\left(D_{0}\right)$, after 15 days of treatment $\left(D_{15}\right)$ and after 30 days of treatment $\left(D_{30}\right)$, with mask PBSK-1 or mask PBSK-2, in 30 human volunteers, comparing each volunteer with its own control at $\mathrm{D}_{0}$. Statistical significances: ${ }^{*} p<0.05 ;{ }^{* *} p<0.01$; and ${ }^{* *} p<0.001$.

Results of crow's feet wrinkles are given in Figure 13. The treatment with mask PBSK-1 significantly decreased length and depth of crow's feet by $8.9 \% \pm 3.2 \%$, and $3.1 \% \pm 0.8 \%$, respectively, whereas the treatment with mask PBSK-2 did not show any statistically significant effect, compared to day 0 . After four weeks of treatment, the outcome showed that treatment with mask PBSK-1 significantly decreased area, length, and depth of crow's feet by $15.4 \% \pm 5.1 \%, 21.9 \% \pm 6.2 \%$, and $5.2 \% \pm 1.0 \%$, respectively. The treatment with mask PBSK-2 did not show any statistically significant effect by comparing each volunteer with its own control at day 0 (Figure 13). Overall, the results of PBSK-1 significantly decreased length of wrinkles after two and four weeks of treatment and depth of wrinkles after four weeks.
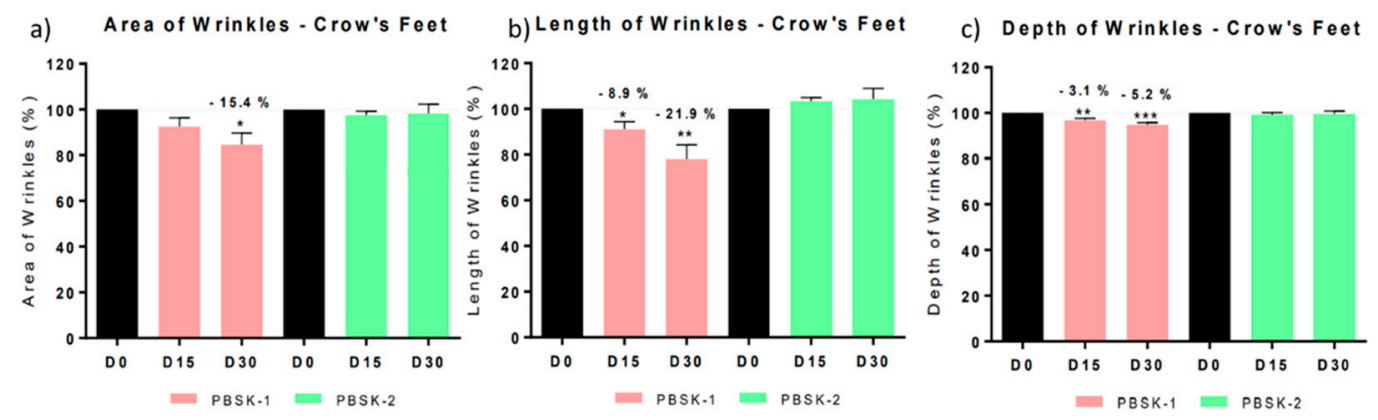

Figure 13. Graphical representation of the total area (a), length (b) and depth (c) of Crow's feet wrinkles before $\left(D_{0}\right)$, after 15 days of treatment $\left(D_{15}\right)$ and after 30 days of treatment $\left(D_{30}\right)$, with mask PBSK-1 or mask PBSK-2, in 30 human volunteers, comparing each volunteer with its own control at day 0. Statistical significances: ${ }^{*} p<0.05 ;{ }^{* *} p<0.01$; and ${ }^{* *} p<0.001$.

The firmness of the skin was also measured during the treatment. Results indicated that treatment with mask PBSK-1 for two weeks significantly increased skin firmness by $5.5 \% \pm 2.2 \%$, compared to day 0 , suggesting the benefits of electrospun pullulan as a skin carrier on its own. Interestingly, the treatment with mask PBSK-2 significantly increased skin firmness by $11.3 \% \pm 3.8 \%$, after 2 weeks of treatment, followed by a non-significant increase after two more weeks of treatment (Figure 14). Comparing results from PBSK-1 against PBSK-2, results indicated the treatment with PBSK-2 significantly decreased skin firmness after four weeks of treatment. 


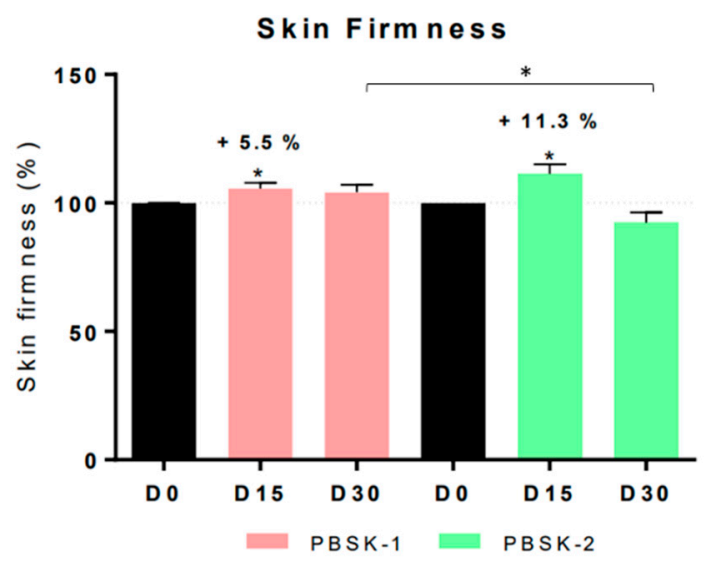

Figure 14. Graphical representation of skin firmness quantified in the skin through Cutometer ${ }^{\circledR}$, before $\left(D_{0}\right)$, after 15 days of treatment $\left(D_{15}\right)$ and after 30 days of treatment $\left(D_{30}\right)$, with mask PBSK-1 or mask PBSK-2, in 30 human volunteers, comparing each volunteer with its own control at day 0 and PBSK-1 with PBSK-2 at the same day. Statistical significances: ${ }^{*} p<0.05$.

Finally, hydration and trans-epidermal water loss (TEWL) were studied. The hydration levels increased for mask PBSK-1 and mask PBSK-2 after two weeks and four weeks of treatment. In fact, a stronger hydration can be seen for mask PBSK-1 with significant hydration levels $59.7 \% \pm 16.1 \%$ and $48.2 \% \pm 10.6 \%$, respectively, after two weeks and four weeks of treatment. In a similar fashion, hydration with mask PBSK-2 at $\mathrm{D}_{15}(17.7 \% \pm 8.0 \%)$ was significantly higher if compared to $\mathrm{D}_{0}$ (Figure $15 \mathrm{a}$ ). This is in good agreement with the hydrophilic nature of pullulan, in which water absorption probably occurred instantaneously.

a)

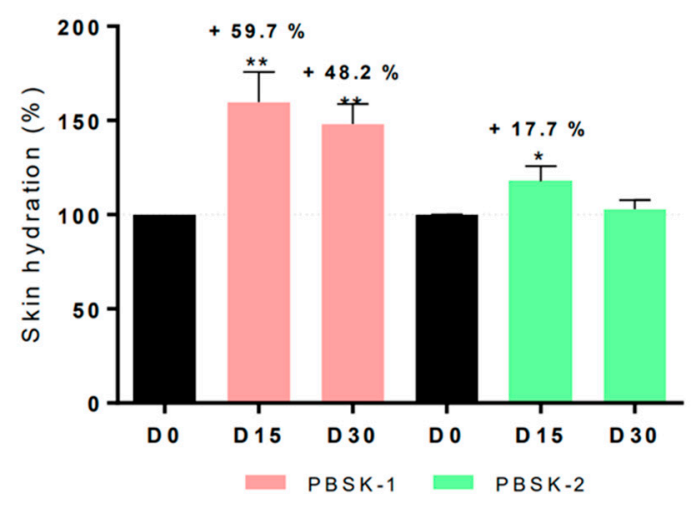

b)

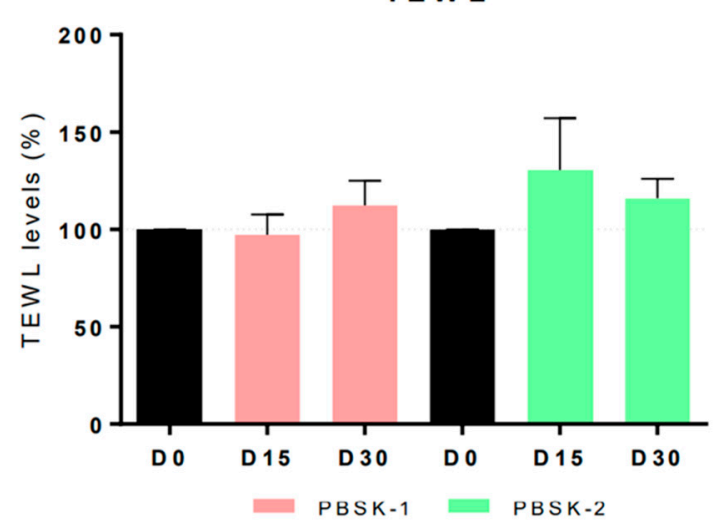

Figure 15. Graphical representation of skin hydration (a) quantified through Corneometer ${ }^{\circledR}$ and TEWL (b) quantified through Tewameter ${ }^{\circledR}$, before $\left(D_{0}\right)$, after 15 days of treatment $\left(D_{15}\right)$ and after 30 days of treatment $\left(\mathrm{D}_{30}\right)$, with mask PBSK-1 or mask PBSK-2, in 30 human volunteers, comparing each volunteer with its own control at day 0 . Statistical significances: ${ }^{*} p<0.05 ;{ }^{* *} p<0.01$.

Concerning the skin barrier function, the treatment with mask PBSK-1 or mask PBSK-2, did not show any significant effect on TEWL levels, after two or four weeks of treatment, compared to basal values at $\mathrm{D}_{0}$, as shown in Figure $15 \mathrm{~b}$.

\section{Discussion}

Cosmetics are very relevant to our society, based on appearance as a means for self-awareness, interaction and communication. Over the past two decades, declining mortality rates have resulted in an increased aged population with a strong desire to maintain a youthful appearance [28]. 
This phenomenon has led to a strong demand for anti-aging products able to prevent wrinkles, age-spots, dry skin and uneven skin tone, among other elderly signs of the skin. The cosmetic industry offers a vast armamentarium of skin care products to take care of the skin across ages. Innovative researches are exploring the use of materials of natural origin and bioactive ingredients with demonstrated antiaging efficacy. Interesting sources have been recently obtained by industrial and agri-food waste with high potential to be used for skin care in sanitary, cosmetic and biomedical sectors [29].

Facial beauty masks represent one of the most largely used cosmetic products. The use of bio-based materials to produce innovative beauty masks aims at combining a green and sustainable approach with skin health and wellbeing, such as protection from oxidative damage and pollution, enable skin microbiota preservation and self-repair capability of the skin [30]. In this study we developed and fully assessed, both in vitro and in vivo, a novel beauty mask fully from natural-origin materials. The beauty mask was produced via electrospinning using a pullulan/water solution and impregnated with micro-complexes derived from nano-sized chitin, lignin and incorporated a licorice compound, GA, which were investigated in a previous study and are referred as CLA [18]. Due to the soluble nature of pullulan, this mask, fabricated as a dry nonwoven, turns into a cream-like consistency upon wetting. The final products can be washed out and dissolved in water.

Over the past decade, electrospinning has become a very popular technology to generate bio-based fibrous materials, especially in the fields of biomedicine, pharmaceutics, and cosmetics [23]. Many natural-occurring materials can be produced to generate fibers via electrospinning [31,32]. Electrospun pullulan fibers have been used in combination with other natural-origin biomaterials and showed good capability of supporting and regulating the cell types involved in skin homeostasis [33]. Under our approach no chemical crosslinking is needed, as the beauty mask will dissolve in water. However, a uniform fiber morphology was required to incorporate CLA. Carriers, such as the pullulan nonwoven, play a critical role in the creation of a cosmetic product by helping to preserve the efficacy, safety and stability of the active ingredients selected. They, in fact, have to load, transport and deliver the actives at the programmed level of the skin, for giving the promised benefits to the customers. The SEM and FTIR-ATR spectroscopy characterization of the beauty masks showed the presence of the impregnated CLA powder in the pullulan electrospun tissue. The peaks attributable to CLA were detected thus highlighting a high CLA content at the mask surface. The load of CLA complexes in the mask was about $12 \%$ by weight. This extremely high load was achieved thanks to the exceptionally low density of the electrospun tissue, which is entitled with high porosity [34]. Having a high loading capacity is fundamental for increasing the beauty mask efficacy. In fact, when the mask is wet, the pullulan tissue is dissolved rapidly thanks to the micrometric dimension of pullulan filaments; hence, the beneficial and abundant CLA complex can be easily and effectively released on the skin. By using HaCaT cells as a model of epidermal response and hMSCs as a model of dermal/hypodermal response, we disclosed the high cytocompatibility of both pullulan and pullulan/CLA beauty masks.

Pullulan beauty mask without CLA was endowed of immunomodulatory properties. Indeed, it was able to upregulate IL-8, IL- 6 and TGF- $\beta$ and to downregulate TNF- $\alpha$, IL- $1 \alpha$ and IL- $1 \beta$. However, it was unable to induce the production of HBD-2. Differently, after dry impregnation with CLA, the beauty mask was able to upregulate all proinflammatory cytokines, and also have a strong indirect antimicrobial activity by a remarkable upregulation of HBD-2. This is due to CLA, and in particular to the presence of CN-NL complex, acting as an inducer of innate immunity, whereas the effect of GA is more related to inflammation modulation [18]. Overall, the in vitro tests suggested that the pullulan/CLA beauty mask may act according to the wound healing process, namely, it promotes an initial raise of the pro-inflammatory cytokines able to stimulate skin regeneration and self-defense.

The in vivo effectiveness was tested with the pullulan/CLA (PBSK-1) fibers and pullulan face mask (PBSK-2). Results indicated that treatment with PBSK-1 significantly displayed antiaging effects, after four weeks of topical application in human volunteers, through significant reduction of forehead and crow's feet wrinkles (area, length, and depth). Moisturizing levels assessed by hydration and 
TEWL indicated that moisturizing levels significantly increased compared to the placebo (PBSK-2), and TEWL remained similar to the values of the skin previous to the treatment, confirming the high tolerability of these beauty masks. Skin firmness was also improved after two weeks of treatment, for both PBSK-1 and PBSK-2. Concerning the anti-irritation effects, after effectively inducing a mild skin irritation on forearms, no statistically significant differences in erythema levels were yielded upon application of the mask PBSK-1 or the mask PBSK-2, at none of the tested timepoints, thus confirming the safety of the product. Regarding dermatological surveillance, the beauty mask showed good skin compatibility and may claim "Dermatologically tested", "Clinically Tested" and "Tolerance Tested". Therefore, the study highlighted that the combination of the hydrophilic nature of pullulan along with the anti-inflammatory and antioxidant properties of the CLA complexes certainly contributed to the improvement in the moisturizing and anti-aging effects, respectively. The pullulan material is a readily water soluble polymer able to solubilize very fast in contact with moisturized skin. This is probably the main factor that contributed to the positive results on moisturizing skin levels. The improvement in wrinkles reduction in such a short timeframe (15-30 days) could be attributed to the efficiently fast release of the CLA from the dissolving mask. The electrospun ultrathin fibers due to their high surface to volume ratio and surface energy stick very efficiently to the inner topology of the skin, delivering the bioactives homogeneously within it, hence presenting an innovative strategy for bioactives delivery in the field of cosmetics.

Bio-based facial masks have been previously studied in an effort to bring to the market innovative bio-friendly solutions. The authors from the study [35] evaluated the efficacy of various wet bio-cellulose masks in vivo to assess the cosmetic effects of anti-aging, lifting and cell renewal. Various solutions were impregnated into the biocellulose carrier to generate three types of masks: anti-aging, lifting and regenerative mask. In this study, a significant decrease in skin roughness and wrinkle breadth was observed after two months treatment with the anti-aging mask [35]. Similarly, an improvement in dermal homogeneity and firmness was also observed after two months with the same type of mask. On the other hand, a significant improvement in skin firmness was observed after one month of treatment with the "lifting" masks. Although promising results were obtained, each prepared beauty presented many different active ingredients and they were wet-based masks. In our study, PBSK-1 itself exerted an anti-aging, lifting (firmness) and moisturizing effect obtaining an all-in-one approach beauty mask. Furthermore, PBSK-1 would only be wetted upon application, thus enhancing product stability.

The possible commercialization of such a dry beauty mask is advantageous to avoid the use of high amount of preservatives, generally included in wet mask formulations. Moreover, as the masks are made fully with bio-based and biodegradable components, their final rinsing from face skin do not determine any significant issue in generating solid waste. This beauty mask is thus representing a smart product with both high performance and a strong ecofriendly profile.

\section{Conclusions}

Thanks to electrospinning and dry powder impregnation technologies it was possible to produce fully skin soluble, bio-based and bioactive pullulan beauty masks incorporating CLA complex with anti-oxidant and anti-inflammatory properties. Due to its extremely low density, the electrospun pullulan tissue was able to incorporate a high content of the bioactive complex in powder, i.e., about $12 \%$ by weight. This high content of CLA incorporated in the pullulan tissue soluble in water granted an efficient methodology for the rapid release of the bioactive complex on the moisturized before application face skin. Our preliminary in vitro study highlighted the wide cytocompatibility of the beauty masks with human cells, used as a model for the skin layers, and suggested an immunostimulatory activity primed by CLA leading to the production of endogenous antimicrobial peptides from the skin. The in vivo topical treatment with the beauty mask (PBSK-1) significantly displayed antiaging effects, after two and four weeks of topical application in human volunteers, through a significant reduction of forehead and crow's feet wrinkles and a significant increase of moisturizing levels, compared to the placebo (PBSK-2), suggesting the effectiveness of the CLA 
complexes combined with the ultrathin fibers of pullulan, as previously exerted in the in vitro studies. On the whole, this work highlights the successful production of an environmentally sustainable and biofunctional cosmetic product, giving novel alternatives to the field of green cosmetics.

Author Contributions: Conceptualization J.M.L.; methodology, J.M.L., S.D., G.D., J.T., M.P.-F., V.B., and N.H.; validation, S.D.,M.-B.C., M.P.F., and J.T.; formal analysis, C.R. and M.P.-F.; investigation, M.P.-F., J.T., N.H., V.B., M.-B.C., S.D., C.R., and A.F.; writing-original draft preparation, M.P.-F. and J.T.; writing-review and editing, J.T., M.P.-F., N.H., M.-B.C., S.D., and J.M.L.; visualization, S.D., G.D., J.T., and M.P.-F.; supervision, S.D., G.D., and J.M.L.; funding acquisition, M.-B.C., S.D., and J.M.L. All authors have read and agreed to the published version of the manuscript.

Funding: This research was funded by the Bio-Based Industries Joint Undertaking under the European Union Horizon 2020 research program (BBI-H2020), PolyBioSkin project, grant number G.A. 745839.

Acknowledgments: Pierfrancesco Morganti (MAVI, Italy) is acknowledged for his precious suggestions regarding cosmetic market trends and clinically correct cosmetics. Karen De Clerck (University of Gent, Belgium) is also thanked for her technical contribution to biopolymers electrospinning. Andrea Lazzeri is thanked for his meaningful discussion regarding nanostructured materials. Finally, Delfo D'Alessandro (University of Pisa, Italy) is greatly acknowledged for his technical support to in vitro experiments.

Conflicts of Interest: The authors declare no conflict of interest in this study.

\section{References}

1. Coltelli, M.-B.; Panariello, L.; Morganti, P.; Danti, S.; Baroni, A.; Lazzeri, A.; Fusco, A.; Donnarumma, G. Skin-Compatible Biobased Beauty Masks Prepared by Extrusion. J. Funct. Biomater. 2020, 11, 23. [CrossRef] [PubMed]

2. Payne, J.; McKeown, P.; Jones, M.D. A circular economy approach to plastic waste. Polym. Degrad. Stab. 2019, 165, 170-181. [CrossRef]

3. Liu, Z.; Anderson, T.D.; Cruz, J.M. Consumer environmental awareness and competition in two-stage supply chains. Eur. J. Oper. Res. 2012, 218, 602-613. [CrossRef]

4. Coltelli, M.-B.; Danti, S.; Trombi, L.; Morganti, P.; Donnarumma, G.; Baroni, A.; Fusco, A.; Lazzeri, A. Preparation of Innovative Skin Compatible Films to Release Polysaccharides for Biobased Beauty Masks. Cosmetics 2018, 5, 70. [CrossRef]

5. Sharif, A.; Akhtar, N.; Khan, M.S.; Menaa, A.; Menaa, B.; Khan, B.A.; Menaa, F. Formulation and evaluation on human skin of a water-in-oil emulsion containing Muscat hamburg black grape seed extract. Int. J. Cosmet. Sci. 2014, 37, 253-258. [CrossRef]

6. Khan, B.A.; Akhtar, N.; Menaa, A.; Menaa, F. A Novel Cassia fistula (L.)-Based Emulsion Elicits Skin Anti-Aging Benefits in Humans. Cosmetics 2015, 2, 368-383. [CrossRef]

7. Coltelli, M.-B.; Danti, S.; De Clerck, K.; Lazzeri, A.; Morganti, P. Pullulan for Advanced Sustainable Bodyand Skin-Contact Applications. J. Funct. Biomater. 2020, 11, 20. [CrossRef]

8. Kumar, D.; Saini, N.; Pandit, V.; Ali, S. An Insight to Pullulan: A Biopolymer in Pharmaceutical Approaches. Int. J. Basic Appl. Sci. 2012, 1, 202-219. [CrossRef]

9. Morganti, P.; Yudin, V.E.; Morganti, G.; Coltelli, M.-B. Trends in Surgical and Beauty Masks for a Cleaner Environment. Cosmetics 2020, 7, 68. [CrossRef]

10. United States Department of Agriculture Agricultural Marketing Service. National Organic Program Document Cover Shee; United States Department of Agriculture Agricultural Marketing Service: Washington, DC, USA, 2018; p. 2544.

11. Pardo-Figuerez, M.; López-Córdoba, A.; Torres-Giner, S.; Lagaron, J.M. Superhydrophobic Bio-Coating Made by Co-Continuous Electrospinning and Electrospraying on Polyethylene Terephthalate Films Proposed as Easy Emptying Transparent Food Packaging. Coatings 2018, 8, 364. [CrossRef]

12. Kadam, V.V.; Wang, L.; Padhye, R. Electrospun nanofibre materials to filter air pollutants-A review. J. Ind. Text. 2016, 47, 2253-2280. [CrossRef]

13. Kim, C. Cosmeticsheet Formed from Nanofiber with Controlled Dissolution Velocity and Method of Manufacturing the Same. U.S. Patent Application 14/437,234, 1 December 2015.

14. Fathi-Azarbayjani, A.; Qun, L.; Chan, Y.W.; Chan, S.Y. Novel Vitamin and Gold-Loaded Nanofiber Facial Mask for Topical Delivery. AAPS PharmSciTech 2010, 11, 1164-1170. [CrossRef] [PubMed] 
15. Morganti, P.; Palombo, M.; Carezzi, F.; Nunziata, M.L.; Morganti, G.; Cardillo, M.; Chianese, A. Green Nanotechnology Serving the Bioeconomy: Natural Beauty Masks to Save the Environment. Cosmetics 2016, 3, 41. [CrossRef]

16. Marduel, J. Apparatus and Method for Mipregnation by Transferringa Powder into Aporous Substrate. U.S. Patent 10,821,471, 3 November 2020.

17. Azimi, B.; Thomas, L.; Fusco, A.; Kalaoglu-Altan, O.I.; Basnett, P.; Cinelli, P.; De Clerck, K.; Roy, I.; Donnarumma, G.; Coltelli, M.-B.; et al. Electrosprayed Chitin Nanofibril/Electrospun Polyhydroxyalkanoate Fiber Mesh as Functional Nonwoven for Skin Application. J. Funct. Biomater. 2020, 11, 62. [CrossRef] [PubMed]

18. Danti, S.; Trombi, L.; Fusco, A.; Azimi, B.; Lazzeri, A.; Morganti, P.; Coltelli, M.-B.; Donnarumma, G. Chitin Nanofibrils and Nanolignin as Functional Agents in Skin Regeneration. Int. J. Mol. Sci. 2019, 20, 2669. [CrossRef] [PubMed]

19. Kowalska, A.; Kalinowska-Lis, U. 18 $\beta$-Glycyrrhetinic acid: Its core biological properties and dermatological applications. Int. J. Cosmet. Sci. 2019, 41, 325-331. [CrossRef]

20. Menaa, F.; Menaa, A.; Tréton, J. Polyphenols against Skin Aging. In Polyphenols in Human Health and Disease; Elsevier BV: Amsterdam, The Netherlands, 2014; pp. 819-830.

21. Khan, B.A.; Akhtar, N.; Menaa, B.; Menaa, A.; Braga, V.A.; Menaa, F. Relative Free Radicals Scavenging and Enzymatic Activities of Hippophae rhamnoides and Cassia fistula Extracts: Importance for Cosmetic, Food and Medicinal Applications. Cosmetics 2017, 4, 3. [CrossRef]

22. Kong, H.; West, S.; States, U. Declaration of Helsinki Ethical Principles for Medical Research Involving Human Subjects; The World Medical Association, Seoul (Korea) Inc.: Seoul, Korea, 2008.

23. Torre-Muruzabal, A.; Daelemans, L.; Van Assche, G.; De Clerck, K.; Rahier, H. Creation of a nanovascular network by electrospun sacrificial nanofibers for self-healing applications and its effect on the flexural properties of the bulk material. Polym. Test. 2016, 54, 78-83. [CrossRef]

24. Cuvellier, A.; Torre-Muruzabal, A.; Kizildag, N.; Daelemans, L.; Ba, Y.; De Clerck, K.; Rahier, H. Coaxial electrospinning of epoxy and amine monomers in a pullulan shell for self-healing nanovascular systems. Polym. Test. 2018, 69, 146-156. [CrossRef]

25. Shingel, K. Determination of structural peculiarities of dexran, pullulan and $\gamma$-irradiated pullulan by Fourier-transform IR spectroscopy. Carbohydr. Res. 2002, 337, 1445-1451. [CrossRef]

26. Li, R.; Tomasula, P.; De Sousa, A.M.M.; Liu, S.-C.; Tunick, M.H.; Liu, Z.; Liu, L.S. Electrospinning Pullulan Fibers from Salt Solutions. Polymers 2017, 9, 32. [CrossRef] [PubMed]

27. Panyamao, P.; Ruksiriwanich, W.; Sirisa-Ard, P.; Charumanee, S. Injectable Thermosensitive Chitosan/Pullulan-Based Hydrogels with Improved Mechanical Properties and Swelling Capacity. Polymers 2020, 12, 2514. [CrossRef] [PubMed]

28. United Nations Department of Economic and Social Affairs. Population Division (2017); United Nations Department of Economic and Social Affairs: New York, NY, USA, 2017; ISBN 9789211515510.

29. Coltelli, M.-B.; Danti, S. Biobased Materials for Skin-Contact Products Promoted by POLYBIOSKIN Project. J. Funct. Biomater. 2020, 11, 77. [CrossRef] [PubMed]

30. Rembiesa, J.; Ruzgas, T.; Engblom, J.; Holefors, A. The Impact of Pollution on Skin and Proper Efficacy Testing for Anti-Pollution Claims. Cosmetics 2018, 5, 4. [CrossRef]

31. Azimi, B.; Maleki, H.; Zavagna, L.; De La Ossa, J.G.; Linari, S.; Lazzeri, A.; Danti, S. Bio-Based Electrospun Fibers for Wound Healing. J. Funct. Biomater. 2020, 11, 67. [CrossRef] [PubMed]

32. Iqbal, H.; Khan, B.A.; Khan, Z.U.; Razzaq, A.; Khan, N.U.; Menaa, B.; Menaa, F. Fabrication, physical characterizations and in vitro antibacterial activity of cefadroxil-loaded chitosan/poly(vinyl alcohol) nanofibers against Staphylococcus aureus clinical isolates. Int. J. Biol. Macromol. 2020, 144, 921-931. [CrossRef]

33. Malafaya, P.B.; Silva, G.A.; Reis, R.L. Natural-origin polymers as carriers and scaffolds for biomolecules and cell delivery in tissue engineering applications. Adv. Drug Deliv. Rev. 2007, 59, 207-233. [CrossRef]

34. Rnjak-Kovacina, J.; Weiss, A. Increasing the Pore Size of Electrospun Scaffolds. Tissue Eng. Part B Rev. 2011, 17, 365-372. [CrossRef] 
35. Perugini, P.; Bleve, M.; Redondi, R.; Cortinovis, F.; Colpani, A. In vivo evaluation of the effectiveness of biocellulose facial masks as active delivery systems to skin. J. Cosmet. Dermatol. 2019, 19, 725-735. [CrossRef]

Publisher's Note: MDPI stays neutral with regard to jurisdictional claims in published maps and institutional affiliations.

(C) 2020 by the authors. Licensee MDPI, Basel, Switzerland. This article is an open access article distributed under the terms and conditions of the Creative Commons Attribution (CC BY) license (http://creativecommons.org/licenses/by/4.0/). 